\title{
STK10 knockout inhibits cell migration and promotes cell proliferation via modulating the activity of ERM and p38 MAPK in prostate cancer cells
}

\author{
LU ZHANG ${ }^{1}$, SHUN-YUAN LU ${ }^{1}$, RUI GUO ${ }^{1}$, JIN-XIA MA ${ }^{1}$, LING-YUN TANG ${ }^{1}$, JIN-JIN WANG ${ }^{2}$, \\ CHUN-LING SHEN ${ }^{1}$, LI-MING LU ${ }^{3}$, JIE LIU ${ }^{4}$, ZHU-GANG WANG ${ }^{1}$ and HONG-XIN ZHANG ${ }^{1}$
}

\author{
${ }^{1}$ Research Center for Experimental Medicine, State Key Laboratory of Medical Genomics, Shanghai Ruijin Hospital, \\ Shanghai Jiao Tong University School of Medicine, Shanghai 200025; ${ }^{2}$ Shanghai Model Organisms Center, Shanghai 201321; \\ ${ }^{3}$ Shanghai Institute of Immunology, Shanghai Jiao Tong University School of Medicine; \\ ${ }^{4}$ Shanghai Institute of Orthopaedics and Traumatology, Shanghai Ruijin Hospital, \\ Shanghai Jiao Tong University School of Medicine, Shanghai 200025, P.R. China
}

Received March 9, 2020; Accepted May 25, 2021

DOI: $10.3892 / \mathrm{etm} .2021 .10283$

\begin{abstract}
Prostate cancer (PCa) is one of the most common types of cancer and is a serious threat to men's health due to the high rate of incidence and metastasis. However, the exact underlying pathology of this malignant disease has yet to be fully elucidated. The ezrin-radixin-moesin (ERM) family of proteins are associated with the development and metastasis of various types of cancer. Serine threonine kinase 10 (STK10) is an ERM kinase that is involved in the activation of ERM proteins and serves essential roles in the aggregation and adhesion of lymphocytes. To evaluate the functional roles of STK10 in the pathogenesis of PCa, a STK10-knockout (KO) DU145 PCa cell line was generated using the CRISPR-Cas9 gene editing system, and the effects of STK10 deletion on tumor biological behaviors were further analyzed. The present data suggested that STK10 KO promoted PCa cell proliferation by inhibiting p38 MAPK activation and suppressed migration primarily via the inhibition of p38 MAPK signaling and ERM protein activation. To the best of our knowledge, this is the first study to provide evidence that STK10 plays important roles in the proliferation and migration of PCa cells, which will be useful for further investigation into the pathogenesis of this disease.
\end{abstract}

Correspondence to: Dr Hong-Xin Zhang or Dr Zhu-Gang Wang, Research Center for Experimental Medicine, State Key Laboratory of Medical Genomics, Shanghai Ruijin Hospital, Shanghai Jiao Tong University School of Medicine, 197 Ruijin Road II, Shanghai 200025, P.R. China

E-mail: zhang_hongxin@hotmail.com

E-mail: redxin678@163.com

Key words: serine threonine kinase 10, ezrin-radixin-moesin, prostate cancer, migration, p38 MAPK

\section{Introduction}

Prostate cancer (PCa) is one of the most common types of malignant cancer affecting the urinary system in men, with $>1$ million new cases diagnosed every year (1). It is a major leading cause of cancer-related mortality, a serious threat to men's health and imposes a heavy economic burden worldwide. PCa generally develops in men aged $\geq 50$ years, with an average age of $\sim 66$ at the time of diagnosis (2). The 5 -year survival rate for most patients with local or regional PCa is $90 \%$ (3). Although significant progress in early diagnosis and clinical therapy of PCa have been achieved in the last several decades, the overall 5 -year survival rate remains $\sim 30 \%$ for men diagnosed with advanced $\mathrm{PCa}$, and metastasis and recurrence are the leading causes of death in patients with PCa (4). The high mortality rate is primarily due to the metastatic spread of tumor cells to lymph nodes, the bladder, bone, spinal cord and some other distant organs (5-7). Therefore, it is necessary to understand the underlying pathogenic mechanisms and to identify novel factors involved in the progression and metastasis of PCa, to assist in identifying novel effective methods for the prevention, diagnosis and therapeutic intervention of this disease.

Cancer metastasis is a multistage process that includes cell invasion, cell migration, colonization, and adaption to and growth in foreign tissues $(8,9)$. At each step of this process, cells undergo morphological changes driven by dynamic remodeling of the actin cytoskeleton (10). Ezrin-radixin-moesin (ERM) proteins are membrane-cytoskeleton linkers that serve critical roles in the regulation of cytoskeleton reorganization and further cell morphological changes, migration and adhesion $(11,12)$. ERM proteins exist in two conformational states, an inactive conformation with the N-terminal FERM domain and C-terminal tail domain (C-ERMAD) forming an intramolecular interaction, and an active conformation where these domains are dissociated. The active conformation of ERM functions as linkers between the cytoskeleton and transmembrane proteins $(13,14)$. Several lines of evidence have 
demonstrated that aberrant localization, expression or activation of ERM proteins are involved in the progression of several types of cancer (15).

The phosphorylation of the FERM domain (T567, T564 and T558, for ezrin, radixin and moesin, respectively) has been identified as the critical step in the activation of ERM proteins $(12,16)$. Serine threonine kinase 10 (STK10), also called lymphocyte-oriented kinase, is a serine/threonine kinase predominantly expressed in lymphoid organs (17). As one of the kinases responsible for the phosphorylation and activation of ERM proteins, STK10 has been demonstrated to be associated with migration and Lymphocyte function-associated antigen-1 (LFA-1)-mediated adhesion of lymphocytes $(18,19)$. Several studies also found that mutations of STK10 are associated with testicular germ cell tumors and aggressive lymphoma. According to the data from The Human Protein Atlas (proteinatlas.org/ENSG00000072786-STK10), STK10 is expressed in 17 types of cancer, including PCa; however, the biological functions of STK10 in the pathogenesis of PCa remains undetermined.

In the present study, to investigate the function of STK10 in the pathology of PCa, an STK10-knockout (KO) DU145 prostate cell line was generated using the CRISPR-Cas9 gene editing system, and the effects of STK10 KO on the tumor biological behaviors, including cell proliferation, apoptosis and migration, were further analyzed. The present data indicated that STK10 KO resulted in decreased migration of PCa cells via regulation of ERM and p38 MAPK activity. These findings uncovered an important role of STK10 in the tumorigenesis of $\mathrm{PCa}$, particularly in cancer metastasis.

\section{Materials and methods}

Cell culture. The PCa cell line (DU145) was purchased from the American Type Culture Collection and grown in RPMI-1640 medium (Hyclone; Cytiva) with 10\% (vol/vol) FBS (Gibco; Thermo Fisher Scientific, Inc.) at $37^{\circ} \mathrm{C}$ with $5 \% \mathrm{CO}_{2}$ in a humidified incubator.

STK10 KO using the CRISPR Cas9 gene editing system. The guide-RNA (5'-GGCGGACGTGCTCATATTCG-3') was designed using the online CRISPR design tool (zlab. bio/guide-design-resources) to target exon 1 of the STK10 gene. Two partially complementary oligonucleotides (5'-CACCGG CGGACGTGCTCATATTCG-3' and 5'-AAACCGAATATG AGCACGTCCGCC-3') were annealed and cloned into the PX459 plasmid (cat. no. 62988; Addgene, Inc.), which was digested using the $B b s I$ restriction enzyme. This plasmid was termed the STK10-KO plasmid, which could guide hSpCas9 to the genomic target site in the STK10 gene. DU145 cells were seeded into a $6-\mathrm{cm}$ dish ( $8 \times 10^{5}$ cells) and transfected with $4 \mu \mathrm{g}$ STK10-KO plasmids or PX459 plasmids (Ctrl) using Lipofectamine ${ }^{\circledR} 3000$ (Thermo Fisher Scientific, Inc.). The supernatant was exchanged for fresh medium $12 \mathrm{~h}$ later. After 2 days, the cells were incubated in fresh medium with $1 \mu \mathrm{g} / \mathrm{ml}$ puromycin for 1 week. Subsequently, the concentration of cells were adjusted to 10 cells/ml by limiting dilution method and the cells were sub-cultured into 96 -well plates (100 $\mu \mathrm{l} /$ well) for single clone selection. Single STK10-KO and control cell clones were identified by western blot analysis and Sanger sequencing using specific primers (forward, 5'-GCCTCGATATTCCCACA GCA-3' and reverse, 5'-CAGGGCACACTTGACCGAG-3'; sequencing primer, 5'-CGGGTCTGGGGAGAACCCCG-3').

STK10 expression vector construction. Expression constructs of STK10 were cloned from cells using the PrimeSTAR ${ }^{\circledR}$ HS PCR kit (cat. no. R040A; Takara Bio, Inc.) with the following primers: forward, 5'-TGCTGGATATCTGCAGAATTCACGCGGC GTCCTCCAACTC-3' and reverse, 5'-TAGTCCAGTGTGG TGGAATTCAGAAGCATCCGCAGAACTGTAGGG-3'. The following temperature protocol was used: $98^{\circ} \mathrm{C}$ for $10 \mathrm{~min}$, followed by 35 cycles of $98^{\circ} \mathrm{C}$ for $10 \mathrm{sec}, 60^{\circ} \mathrm{C}$ for $10 \mathrm{sec}$ and $72^{\circ} \mathrm{C}$ for $3 \mathrm{~min}$; then $72^{\circ} \mathrm{C}$ for $10 \mathrm{~min}$. STK10 fragment was cloned into the pcDNA3.1 myc-His(-) B plasmid (cat. no. V855-20; Thermo Fisher Scientific, Inc.). Final constructs contained the affinity tags Myc and His, and were transcribed under the control of the CMV promoter.

Western blot analysis. DU145 cells were grown in 60-mm dishes and then cultured in serum-free RPMI-1640 medium for $6 \mathrm{~h}$, following which, they were stimulated with $50 \mathrm{ng} / \mathrm{ml}$ human recombinant epidermal growth factor (EGF; R\&D Systems, Inc.) for the appropriate times $(5,15$ and $30 \mathrm{~min}$ ) at $37^{\circ} \mathrm{C}$ with $5 \% \mathrm{CO}_{2}$ in a humidified incubator. DU145 cells were grown in a six-well plate and then transfected with the indicated STK10 expression vectors or empty vectors using Lipofectamine ${ }^{\circledR} 3000$, according to the manufacturer's instructions. Cells were collected $48 \mathrm{~h}$ after transfection. STK10-KO DU145 and control cell lysates were prepared using RIPA lysis buffer [1\% Nonidet P-40, $0.5 \%$ sodium deoxycholate, $0.1 \%$ SDS in phosphate-buffered saline (PBS)] with freshly supplemented protease inhibitors cocktail (Roche Diagnostics). The concentration of proteins was detected using a bicinchoninic acid assay, and $40 \mu \mathrm{g}$ protein was separated using 10\% SDS-PAGE. Standard protocols were used for electrophoresis and immunoblotting analyses (20). Membranes were blocked with 5\% milk in PBS for $1 \mathrm{~h}$ at room temperature. An anti-STK10 antibody (dilution 1:1,000; cat. no. ab70484; Abcam), anti-GAPDH rabbit polyclonal antibody (dilution 1:2,000; cat. no. D110016; BBI Solutions), recombinant anti-Ezrin antibody (dilution 1:500; cat. no. ab40839; Abcam), recombinant anti-Moesin antibody (dilution 1:500; cat. no. ab52490; Abcam), recombinant anti-Radixin antibody (dilution 1:1,000; cat. no. ab52495; Abcam), anti-Ezrin (pThr567)/Radixin (pThr564)/Moesin (pThr558) antibody (dilution 1:500; cat. no. ab76247; Abcam), anti-p38 MAPK antibody (dilution 1:1,000; cat. no. 9212; Cell Signaling Technology, Inc.), anti-phospho-p38 MAPK antibody (dilution 1:1,000; cat. no. 9211; Cell Signaling Technology, Inc.), anti-ERK1/2 antibody (dilution 1:1,000; cat. no. 9102; Cell Signaling Technology, Inc.), anti-phospho-ERK1/2 antibody (dilution 1:1,000; cat. no. 4370; Cell Signaling Technology, Inc.), anti-phospho-PI3K p85 antibody (dilution 1:1,000; cat. no. 4228; Cell Signaling Technology, Inc.), anti-PI3K p85 antibody (dilution 1:1,000; cat. no. 4292; Cell Signaling Technology, Inc.), anti-PI3K, phosphatidylinositol-4,5-bisphosphate 3-kinase, catalytic subunit (p110) $\alpha$ (dilution 1:1,000; cat. no. 4249; Cell Signaling Technology, Inc.), anti-PI3K p110 $\gamma$ (dilution 1:1,000; cat. no. 5405; Cell Signaling Technology, Inc.), anti-Cyclin D1 (dilution 1:500; cat. no. 2978; Cell 
Signaling Technology, Inc.), anti-Cyclin D3 (dilution 1:1,000; cat. no. 2936; Cell Signaling Technology, Inc.), anti-zinc finger E-box binding homeobox 1 (dilution 1:500; ZEB1; cat. no. 3396; Cell Signaling Technology,Inc.) and anti-E-Cadherin (dilution 1:1,000; cat. no. 3195; Cell Signaling Technology, Inc.) were used as the primary antibodies and incubated overnight at $4^{\circ} \mathrm{C}$. Subsequently, the membranes were incubated with IRDyeCW800-conjugated anti-rabbit immunoglobulin (dilution 1:10,000; cat. no. 926-32213; LI-COR Biosciences, Inc.). The images were captured using the LI-COR Odyssey imaging system (LI-COR Odyssey 9120 imaging system; LI-COR Biosciences, Inc.) and adjusted to grayscale images according to the fluorescence intensity of the bands.

Immunofluorescence staining. DU145 cells grown on coverslips $\left(8 \times 10^{4}\right.$ cells per well in a 12 -well plate) for $48 \mathrm{~h}$ were fixed with 4\% paraformaldehyde in PBS for $10 \mathrm{~min}$ at room temperature and washed with PBS three times. To permeabilize the cells, $0.5 \%$ Triton X-100 in PBS-Tween (PBST) was used to permeabilize cells for $10 \mathrm{~min}$ at room temperature. Coverslips were incubated with $10 \%$ goat serum (Gibco; Thermo Fisher Scientific, Inc.) in PBST for 30 min to block non-specific antibody binding. An anti-STK10 antibody (1:500; cat. no. ab70484; Abcam) was used as the primary antibody and incubated overnight at $4^{\circ} \mathrm{C}$. Then, the coverslips were washed three times with PBST. Cells were incubated with the goat anti-rabbit IgG $(\mathrm{H}+\mathrm{L})$ Highly Cross-Adsorbed secondary antibody-Alexa Fluor Plus 594 (1:500; cat. no. A32740; Thermo Fisher Scientific, Inc.) for $2 \mathrm{~h}$ at room temperature. Coverslips were washed three times with PBST, and incubated with DAPI for $10 \mathrm{~min}$ at room temperature. Coverslips were mounted using Fluorescence Mounting Medium (Dako; Agilent Technologies, Inc.) and imaged by fluorescence microscopy (magnification, x400; Nikon Corporation).

Cell Counting Kit-8 (CCK-8) cell viability assay. Cell viability was detected using a CCK-8 assay (Dojindo Molecular Technologies, Inc.), according to the manufacturer's instructions. DU145 cells were seeded into a 96-well plate with $1 \times 10^{3}$ cells/well. Viability was detected following incubation of the cells with $10 \%$ CCK- 8 solution in complete medium for $24 \mathrm{~h}$. The absorbance was detected at $450 \mathrm{~nm}$ using a microplate reader (BioTek Instruments, Inc.).

BrdU incorporation and apoptosis detection. Cell proliferation and the cell cycle were analyzed using a Phase-Flow ${ }^{\text {TM }}$ FITC BrdU kit (BioLegend, Inc.) following the manufacturer's instructions. First, BrdU solution $(5 \mu \mathrm{g} / \mathrm{ml})$ was added to the cell suspension and cultured for $1 \mathrm{~h}$. Then, the cells were dyed with an anti-BrdU antibody and 7-AAD, and analyzed by flow cytometry (BD FACSVerse ${ }^{\mathrm{TM}}$; BD Biosciences, Inc.). To detect cell apoptosis, an Annexin V-FITC Apoptosis Detection kit (eBioscience; Thermo Fisher Scientific, Inc.) according to the manufacturer's protocol. The results were analyzed using software FlowJo version 10 (FlowJo LLC).

Cell migration assay. Cells were starved in serum-free medium for $24 \mathrm{~h}$, Transwell chambers with an $8 \mu \mathrm{m}$ pore size (EMD Millipore) were inserted into a 24 -well culture plate. DU145 cells $\left(1 \times 10^{5}\right)$ were resuspended in $400 \mu 1$ serum-free medium and added to the upper chamber. Complete medium (600 $\mu \mathrm{l})$ was added to the outside of the inserts and incubated at $37^{\circ} \mathrm{C}$ in a cell incubator for $48 \mathrm{~h}$. Cells were fixed with $4 \%$ paraformaldehyde for $10 \mathrm{~min}$ at room temperature and stained with $0.5 \%$ crystal violet for $5 \mathrm{~min}$ at room temperature. Cells which had not migrated were removed using a cotton swab. Cells which had migrated were imaged using a light microscope (magnification, x100). The number of cells in five random fields of view were counted, and the mean was calculated.

In vivo mice study. Male NPSG immune deficient mice (NOD-Prkdc ${ }^{\text {scid }} I l 2$ rg $^{\text {null }}$, weight: 19.46-22.23 g; 4-weeks old) were injected with DU145 cells $\left(1 \times 10^{6}\right)$ in $100 \mu$ l medium mixed with Matrigel (ratio 1:1; Invitrogen; Thermo Fisher Scientific, Inc.) in the flank regions. All mice were housed with a 12-h light/dark cycle, with ad libitum access to food and water. Adequate humanitarian care was provided. For this study, the endpoints were a tumor burden $>10 \%$ of body weight, any tumor $>20 \mathrm{~mm}$ in diameter, ulceration, necrosis or infection, and the presence of a tumor that interfered with eating or walking in an adult mouse. A total of 8 mice were used in this experiment, and none of them reached the endpoint of the study. After 29 days, all mice were euthanized with $\mathrm{CO}_{2}$, before the longest dimension of the tumors reached $20 \mathrm{~mm}$. Initial $\mathrm{CO}_{2}$ delivery to the micro-isolator was accomplished by opening the $\mathrm{CO}_{2}$ cylinder valve such that animals were slowly exposed to increasing levels of $\mathrm{CO}_{2}$ (displacing $\sim 40 \%$ of the chamber volume per min). Cardiac arrest for $5 \mathrm{~min}$ was used to confirm death. Tumor tissues were collected and weighed. Tumors were measured using a digital caliper, and the tumor volume was calculated as follows: (width) ${ }^{2} \mathrm{x}$ length/2. All experimental manipulations were approved by the Animal Ethics Committee of Ruijin Hospital Affiliated to Shanghai Jiao Tong University School of Medicine (Shanghai, China).

Statistical analysis. Each experiment was performed at least three times independently, and results are presented as the mean \pm standard deviation. All statistical analyses were performed using GraphPad Prism version 7 (GraphPad Software, Inc.). Comparisons between two groups were performed using an unpaired Student's t-test. $\mathrm{P}<0.05$ was considered to indicate a statistically significant difference.

\section{Results}

Generation of STK10-KO cell lines using the CRISPR-Cas9 gene editing system. The CRISPR-Cas9 gene editing system was used to generate a STK10-KO PCa cell line. The oligonucleotides for the guide RNA were designed, synthesized and cloned into the pX459 vector (Fig. 1A). Then, the vectors were transfected into DU145 cells. The indel mutations in these cell lines were confirmed by DNA sequencing of the PCR products of target DNA. The insertion of one base (c.66_67insA) causes frameshift mutation, resulting in the formation of a stop codon and deficiency of the STK10 protein (Fig. 1B). Finally, western blotting and immunofluorescence staining with an anti-STK10 antibody showed that the expression of STK10 protein was abolished in the STK10-KO cell line (Fig. 1C and D). 
A

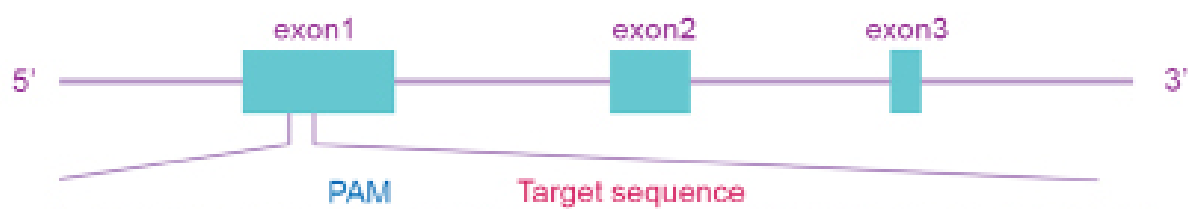

5. AGAGAAAGTCCCGCGAATATGAGCACGTCCGCCGCGACCTGGA 3 '

3' TCTCTTTCAGGGCGCTTATACTCGTGCAGGCGGCGCTGGACCT 5'

Cas 9 cut site

B
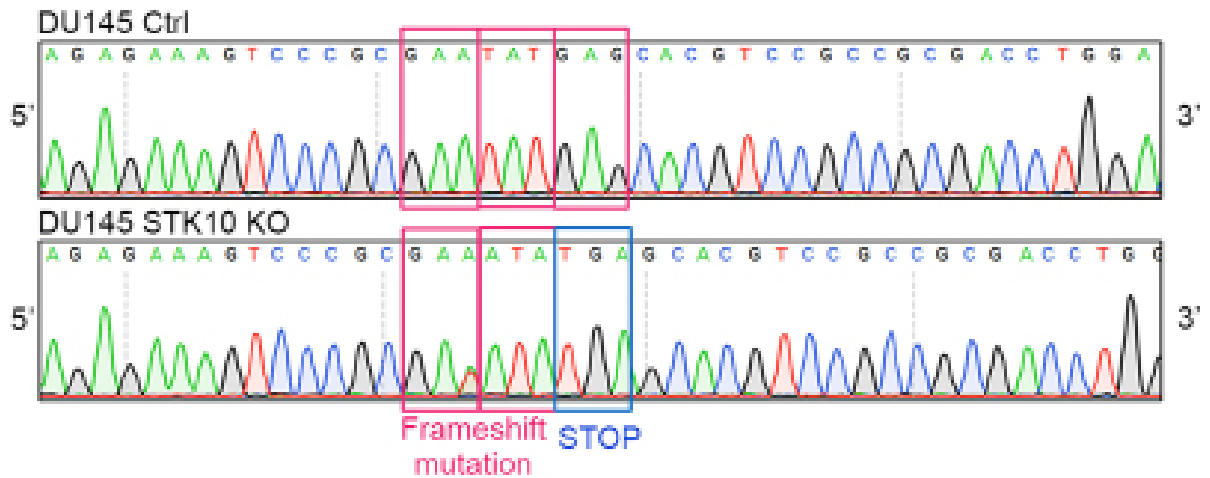

C

D
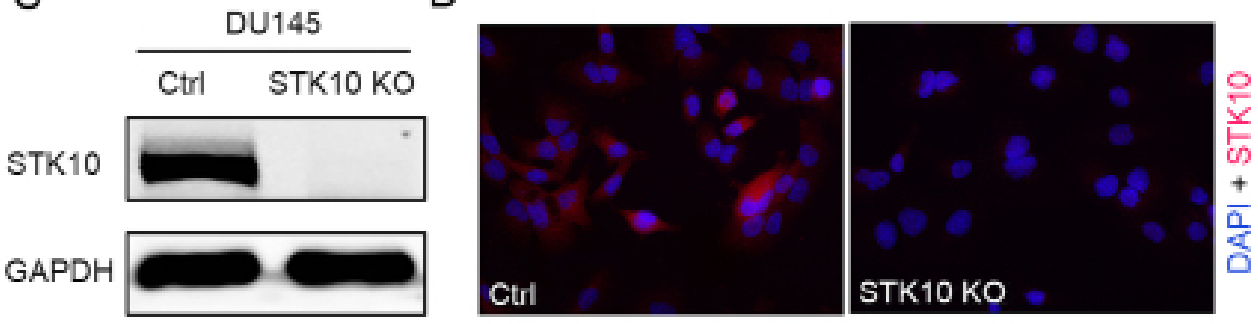

Figure 1. Generation and validation of STK10-KO PCa cell lines. (A) Gene targeting strategy for generating STK10-KO DU145 PCa cells. (B) DNA sequence analysis showed the presence of the STK10 mutation in DU145 cells. (C) Western blot analysis of STK10-KO DU145 cells. (D) Immunofluorescence staining of STK10 in control and STK10-KO DU145 cells. STK10, serine threonine kinase 10; KO, knockout; PCa, prostate cancer; Ctrl, control.

Target deletion of STK10 in PCa cells promotes proliferation but suppresses migration. The effects of STK10 deficiency on the spontaneous proliferation of PCa cells was first evaluated using a BrdU incorporation assay and cell cycle distribution was assessed using fluorescence activated cell sorting (FACS). The results indicated that target deletion of STK10 markedly decreased the percentage of cells in the G0/G1 phase $(46.23 \pm 1.77$ vs. $62.77 \pm 2.01 \%$; $\mathrm{P}<0.001)$, but increased the proportion of cells in the $\mathrm{S}$ phase $(19.03 \pm 0.61$ vs. $11.40 \pm 0.60 \% ; \mathrm{P}<0.001)$ and $\mathrm{G} 2$ phase $(20.07 \pm 1.78$ vs. $15.37 \pm 1.40 \% ; \mathrm{P}<0.01)$ in DU145 $\mathrm{PCa}$ cells (Fig. 2A and D). Furthermore, a CCK-8 assay was performed for 1-4 days to evaluate the proliferation of DU145 control and STK10-KO cells. As shown in Fig. 2C, the cell viability and proliferative capacity of STK10-KO DU145 cells was significantly increased when compared with the control cells. These data suggest that the KO of STK10 promoted cell cycle progression of PCa cells.

Since abnormal apoptosis is one of the most important characteristics of malignant cancer cells, Annexin V-PI staining and flow cytometry analysis was performed to further evaluate the impact of STK10 on the apoptosis of PCa cells. Compared with the control cells, the percentage of early apoptotic cells (Annexin $\mathrm{V}^{+} / \mathrm{PI}^{-}, 1.36 \pm 0.39$ vs. $3.93 \pm 0.64 \%$; $\mathrm{P}<0.01$ ) and late apoptotic cells (Annexin $\mathrm{V}^{+} / \mathrm{PI}^{+}, 0.43 \pm 0.03$ vs. $0.76 \pm 0.05 \%$;
$\mathrm{P}<0.001)$ were decreased in STK10-KO cells (Fig. 2B and E). However, these low percentages $(<10 \%)$ of apoptotic rates are not physiologically significant in vitro (21). These results suggested that STK10 KO has no significant biological effects on the apoptosis of PCa cells.

To explore the role of STK10 on the tumorigenesis and metastasis of PCa, the migratory ability of DU145 PCa cells was detected in vitro. The Transwell assays showed that the number of cells that had migrated were decreased in the STK10-KO group compared with that in the control group $(\mathrm{P}<0.0001$; Fig. 2F and G). These results demonstrated that knockdown of STK10 could inhibit the migration of PCa cells, and indicated that STK10 may serve an essential role in the metastasis of PCa.

Target deletion of STK10 facilitates the growth of tumor xenografts in vivo. After it was determined that STK10 deletion promoted the proliferation and suppressed the apoptosis of PCa cells in vitro, the effect of STK10 KO on xenograft tumors in immunodeficient mice were investigated. As shown in Fig. 3A and B, the tumor weights were significantly increased in mice bearing DU145 STK10-KO cells $(0.805 \pm 0.278$ vs. $0.354 \pm 0.122 \mathrm{~g} ; \mathrm{P}<0.05)$. Tumor progression was more rapid in the DU145 STK10-KO group than those inoculated with DU145 control cells (Fig. 3C). The difference 
A

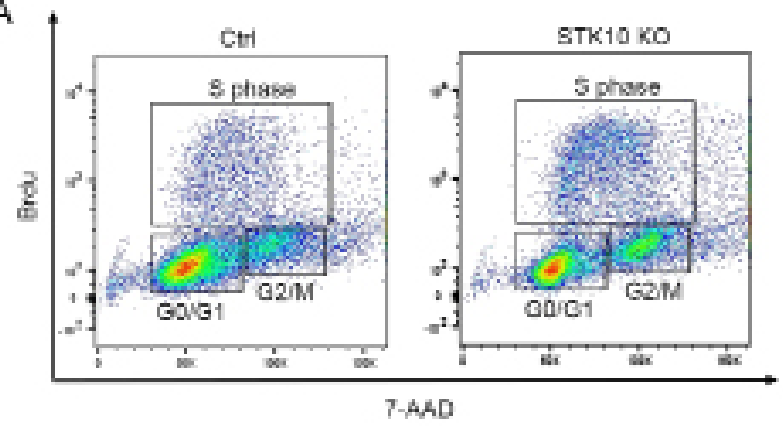

B

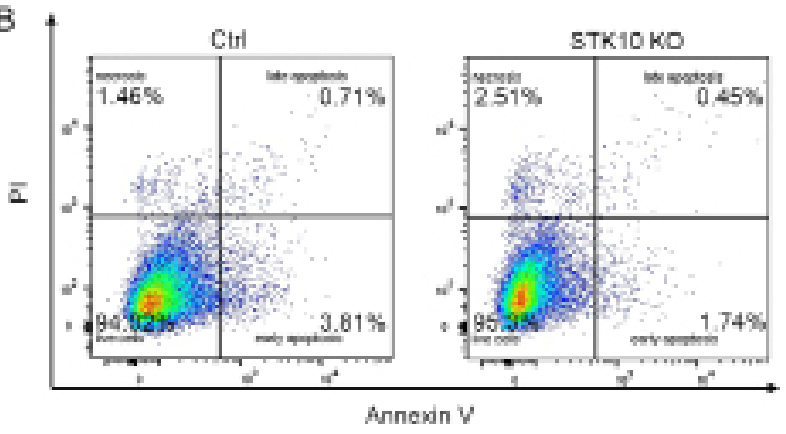

E

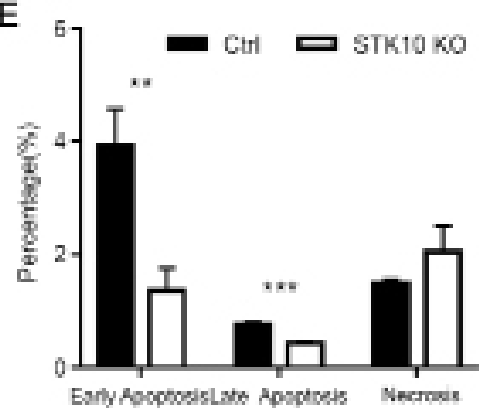

G

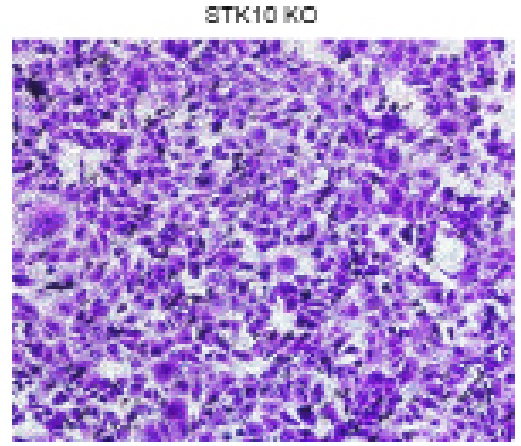

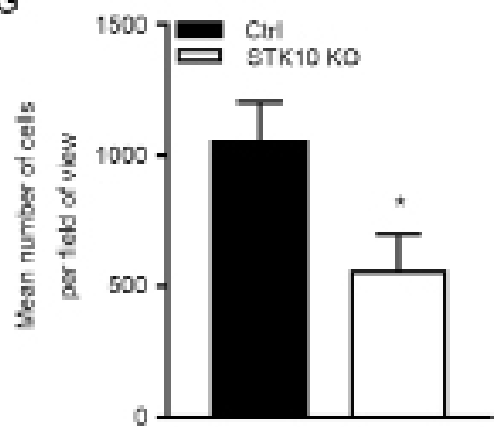

Figure 2. Effects of STK10 KO on the proliferation, apoptosis and migration of PCa cells. (A) BrdU incorporation and FACS analyses for control and STK10-KO DU145 cells. (B) Effects of STK10 KO on the apoptosis of DU145 cells were examined by Annexin V-FITC/PI staining. (C) Proliferative activity of control and STK10-KO DU145 cells was quantified using a Cell Counting Kit-8 assay. (D and E) The percentages of different stages of the cell cycle and apoptotic cells were calculated. $n=3$ in each group. All experiments were performed at least three times. $(\mathrm{F}$ and $\mathrm{G})$ Representative images and quantitative analysis of the Transwell migration assays. Magnification, x100. Data are presented as the mean \pm standard deviation. ${ }^{*} \mathrm{P}<0.05,{ }^{* *} \mathrm{P}<0.01,{ }^{* * * *} \mathrm{P}<0.001$ vs. Ctrl. STK10, serine threonine kinase 10; KO, knockout; PCa, prostate cancer; FACS, fluorescence activated cell sorting; AAD, 7-amino actinomyosin D; Ctrl, control.

in tumor volume was significant after 22 days of subcutaneous injection, especially on days 25 and $29(\mathrm{P}<0.01)$. These results demonstrated that STK10 deletion facilitated tumorigenesis in a xenograft tumor model in vivo.

Disruption of STK10 downregulates the phosphorylation of p38 MAPK and ERM, and influences the expression levels of proteins related to cell migration and proliferation in PCa cells. To investigate the underlying mechanism through which STK10 regulates the biological characteristics of PCa cells, the expression and phosphorylation levels of ERM proteins were determined, as STK10 is one of the kinases responsible for the phosphorylation of ERM proteins (18). The results of western blotting showed that the phosphorylation levels of the ERM-family of proteins were significantly decreased in STK10-KO cells compared with that in the control cells (Fig. 4A). Numerous studies have demonstrated that MAPKs are critical regulators of the survival and migration of cancer cells. Next, the effects of STK10 deletion on the modulation of ERK-MAPK, PI3K and p38 MAPK pathways were assessed. Amongst all the MAPK signaling proteins that were tested in this study, the levels of phosphorylated p38 MAPK was notably decreased following targeted deletion of STK10, whereas the levels of PI3K and phosphorylated ERK were mostly unaffected (Fig. 4A). Since STK10 KO promoted cell proliferation, but inhibited cell migration, the expression levels of proteins that are closely related to cell proliferation and migration were then examined (Fig. 4A). The results showed that Cyclin D1 and Cyclin D3, which promote cell cycle progression, were upregulated in STK10-KO cells. However, the transcription factor ZEB1 was downregulated in STK10-KO cells, which has been demonstrated to have a significant effect on the expression levels of E-Cadherin proteins in numerous studies (22). Thus, compared with the control cells, STK10-KO cells exhibited higher E-Cadherin protein expression levels, which may have effects on the migratory ability of cells. 
A

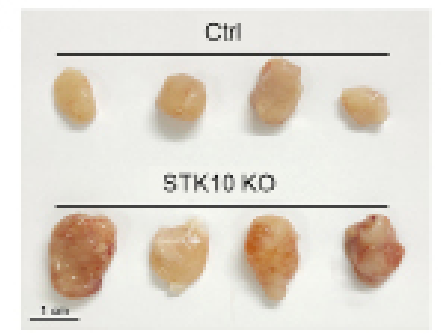

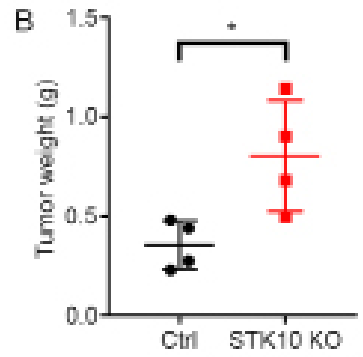

$\mathrm{C}$

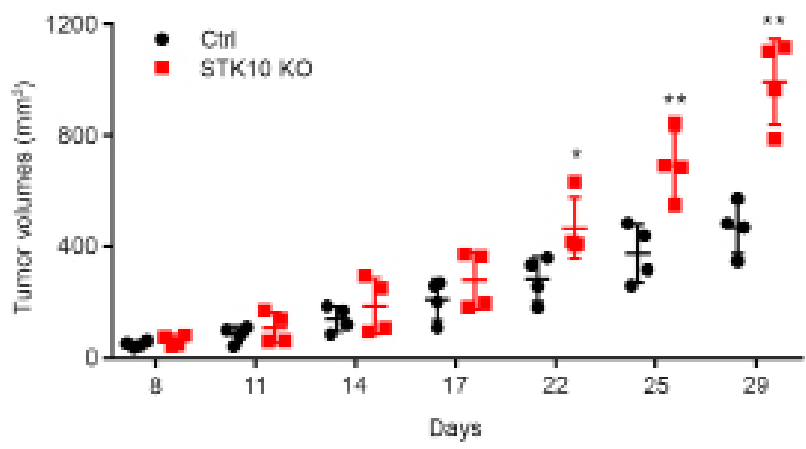

Figure 3. STK10-KO DU145 cells exhibit increased in vivo tumor growth (A) Representative xenograft tumors in each group are shown. Scale bar, $1 \mathrm{~cm}$. (B) Tumor weights are shown in the scatter plot. (C) Tumor sizes were measured on the days indicated and the tumor volumes were calculated. Data are presented as the mean \pm standard deviation. $\mathrm{n}=4$ per group. ${ }^{*} \mathrm{P}<0.05$ ${ }^{* *} \mathrm{P}<0.01$ vs. Ctrl. STK10, serine threonine kinase 10; KO, knockout; Ctrl, control.

It is well known that EGFR and its downstream pathways are associated with the pathological development and progression of PCa. As shown in Fig. 4C, DU145 cells were treated with EGF (50 ng/ml) for 0, 5, 15 and $30 \mathrm{~min}$. The phosphorylation levels of p38 MAPK and ERM increased and decreased as the time changed. However, phosphorylation levels of p38 MAPK and ERM in STK10-KO cells were consistently lower than that in the control cells, and the time required to reach peak ERM phosphorylation was delayed. To further explore the relationship between STK10 and p38, overexpression experiments were conducted. Firstly, 0, 1, 2 or $3 \mu \mathrm{g}$ of the STK10 expression vectors were transfected into DU145 wild-type cells, and the phosphorylation levels of p38 MAPK and ERM were determined using western blotting. The results showed that the phosphorylation levels of p38 MAPK and ERM increased with the increase in STK10 protein expression levels (Fig. 4B). Next, an STK10 expression vector (3 $\mu \mathrm{g})$ or an empty vector were transfected into DU145 control cells and STK10-KO cells. The results showed that when STK10 protein was expressed in STK10-KO cells, phosphorylation levels of p38 MAPK and ERM increased (Fig. 4D). These data indicated that the changes seen in STK10-KO DU145 PCa cells may be due to dysregulated activation of the p38 MAPK and ERM proteins signaling pathways.

\section{Discussion}

Being an extremely heterogeneous malignant disease, $\mathrm{PCa}$ is characterized by abnormal proliferation of cells in the prostate gland and metastasis of tumor cells to other tissues. It has been demonstrated that age, dietary habits, family history, race and some genetic factors are associated with the etiology of PCa (23). However, the exact pathogenesis of this disease is still not fully understood. Therefore, it is necessary to identify novel risk factors and clarify the underlying molecular mechanisms involved in the development and progression of PCa.

In the present study, the role of STK10 in the regulation of the biological characteristics of PCa were assessed using a CRISPR-Cas9-mediated STK10-KO DU145 cell line. The proliferation, cell cycle progression, apoptosis, migration and tumorigenic activity in these cells were detected using CCK-8 and BrdU incorporation assays, Annexin V/PI staining, a Transwell assay and a xenograft tumor model in vivo. The results showed that STK10 depletion promoted proliferation and cell cycle progression, and facilitated the growth of tumor xenografts in vivo; however, it inhibited the apoptosis and migration of PCa cells. Next, the phosphorylation and expression levels of ERM proteins, which are substrates of STK10, were determined. As cytoskeleton-plasma membrane linker proteins, ERM proteins are essential in the metastasis of various types of cancer via regulation of cell morphogenesis, cell survival, cell motility and signaling transduction $(11,15,24,25)$. The present data showed that STK10 KO resulted in decreased ERM phosphorylation, which indicated that STK10 is involved in the migration of PCa cells partially through regulating the activity of ERM proteins.

Given the widespread role of MAPK signaling pathways in the cellular functions of cancer cells, the expression and phosphorylation levels of ERK-MAPK, PI3K and p38 MAPK were detected. Western blotting revealed that STK10 knockdown did not affect the expression and activation of ERK or PI3K, but significantly suppressed the activation of p38 MAPK. A wide range of studies have demonstrated that p38 MAPK signaling inhibits cell proliferation and promotes cell migration in various malignant types of cancer (26-28).

ZEB1 is an epithelial-mesenchymal-transition (EMT)-related transcription factor that is located downstream of the MAPK pathway; it can increase EMT progression in PCa (29). ZEB1 can downregulate E-Cadherin levels and promote cancer invasion (22). Cyclin D1 and Cyclin D3 encode the regulatory subunit of a holoenzyme and promote progression through the G1-S phase of the cell cycle, which serve pivotal roles in the development of a subset of cancers, including PCa, and are regulated by p38 and other kinases (30-32). The results of the present study showed that the protein expression levels of E-Cadherin, Cyclin D1 and Cyclin D3 were upregulated in STK10-KO cells, whereas the transcription factor ZEB1 was downregulated in STK10-KO cells. Thus, the changes in cell proliferation in vitro, tumorigenesis in vivo and migration of STK10-KO DU145 PCa cells, could be attributed to the low activity of p38 MAPK. Although several reports have revealed that p38 MAPK is indirectly implicated in the phosphorylation of ERM proteins $(33,34)$, the relationship between p38 MAPK and ERM kinase STK10 remains to be further elucidated. Nonetheless, the present study demonstrated a novel role of STK10 in the regulation of the p38 MAPK signaling pathway.

Taken together, these data suggest that STK10 KO promotes PCa cell proliferation by inhibiting p38 MAPK 

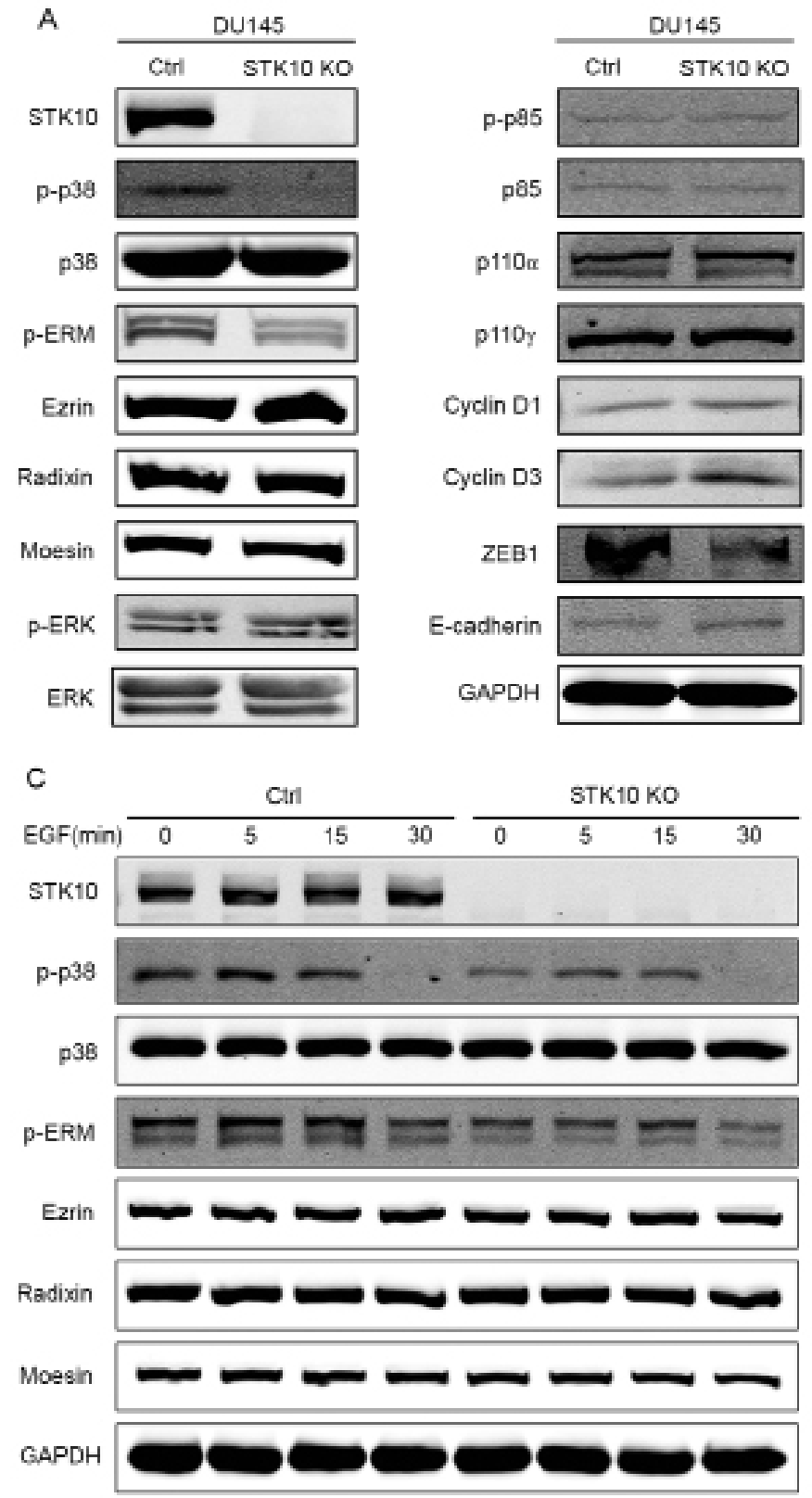

E-cadherin

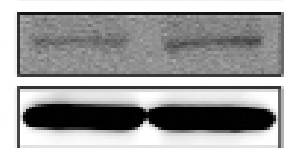

GAPDH
B

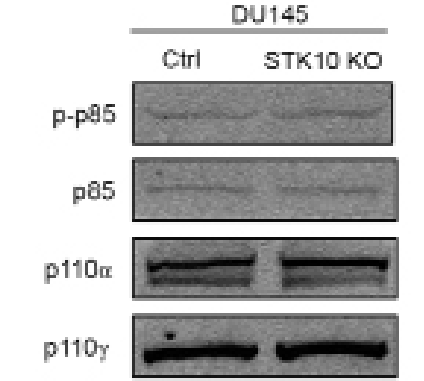

D
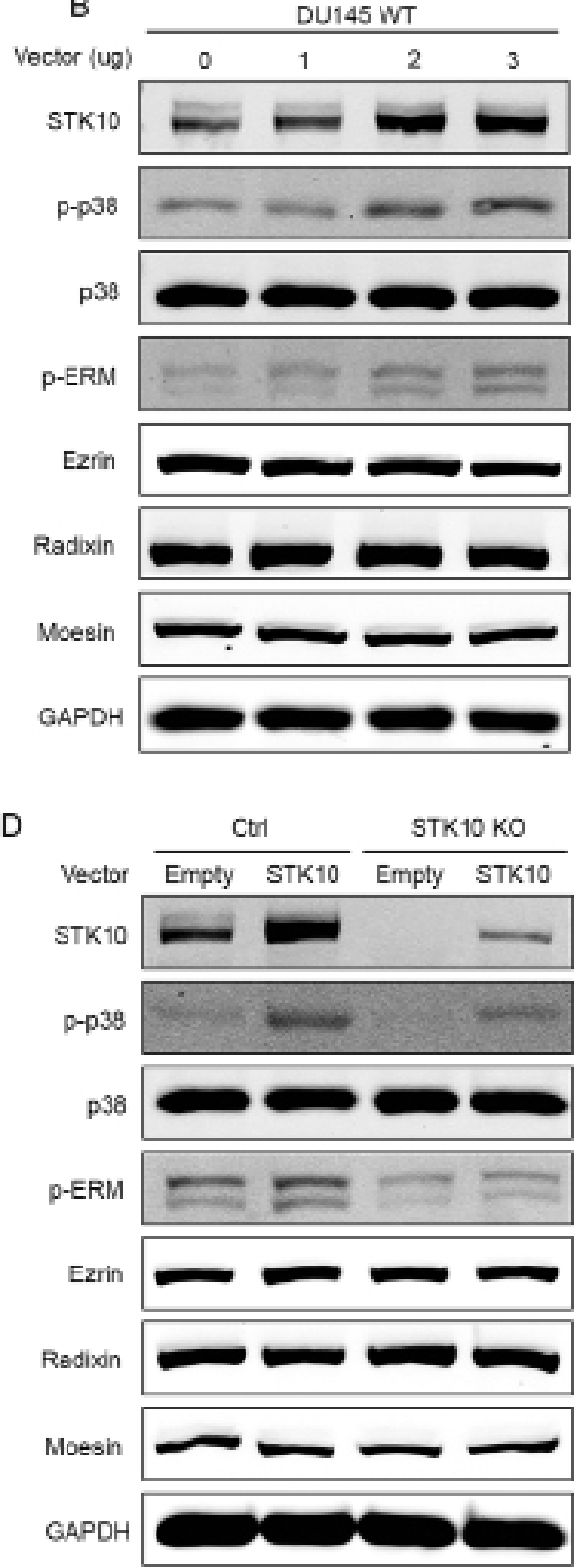

Figure 4. STK10 KO downregulates the phosphorylation level of p38 MAPK and ERM. (A) Western blot analysis showed decreased p38 MAPK and ERM phosphorylation, and increased levels of ZEB1, E-Cadherin, Cyclin D1 and Cyclin D3, but no change in activation of ERK or PI3K in the STK10-KO DU145 cells. (B) DU145 wild-type cells were transfected with the indicated quantity of $S T K 10$ expression vectors $(0,1,2$ and $3 \mu \mathrm{g})$ or empty vectors (the total quantity of vectors was $3 \mu \mathrm{g}$ ). The phosphorylation levels of p38 MAPK and ERM were assayed using western blotting. (C) DU145 control and STK10-KO cells were treated with EGF $(50 \mathrm{ng} / \mathrm{ml})$ for the indicated times $(0,5,15$ and $30 \mathrm{~min})$. The phosphorylation levels of p38 MAPK and ERM were assayed using western blotting. (D) DU145 control and STK10-KO cells were transfected with $3 \mu \mathrm{g}$ STK10 expression vectors or empty vectors. The phosphorylation levels of p38 MAPK and ERM were assayed using western blotting. STK10, serine threonine kinase 10; KO, knockout; ERM, ezrin-radixin-moesin; ZEB1, zinc finger E-box binding homeobox 1; EGF, epidermal growth factor; p-, phospho-; ZEB, zinc finger E-box-binding homeobox 1; p110, phosphatidylinositol-4,5-bisphosphate 3-kinase, catalytic subunit.

activation, and suppresses PCa cell migration mainly via inhibiting P38 MAPK signaling and ERM protein activation. A limitation of this study is the use of only one cell line. The conclusions drawn would be more convincing if similar results were observed in different PCa cell lines. Although more precise studies are required to clarify the functional role of STK10 in the pathogenesis of PCa, to the best of our knowledge, the present study is the first to demonstrate a possible role of STK10 in the proliferation and metastasis of $\mathrm{PCa}$, and provides novel information that may be useful for the prevention, diagnosis and treatment of this disease. In addition, this information may prove to be important in elucidating the biology underlying PCa, which will be of great benefit to patients in the future.

\section{Acknowledgements}

Not applicable.

\section{Funding}

This work was supported by grants from the National Natural Science Foundation of China (grant nos. 81671538 and 81971462), the Shanghai Municipal Bureau of Health for 
researchers (grant nos. 201740191 and 20174Y0120) and the grant from Science and Technology Commission of Shanghai Municipality (grant no. 18ZR1423500).

\section{Availability of data and materials}

The datasets used and/or analyzed during the present study are available from the corresponding author on reasonable request.

\section{Authors' contributions}

LZ and HZ conceived and designed the study. LZ, RG, JM, JW and CS performed the experiments. SL and LT generated cell lines. LL, ZW, JL and HZ analyzed data. JL and HZ wrote the paper. $\mathrm{ZW}$ and $\mathrm{HZ}$ were responsible for research supervision, coordination and strategy. LZ and HZ confirm the authenticity of all the raw data. All authors have read and approved the final manuscript.

\section{Ethics approval and consent to participate}

All experimental manipulations were approved by the Animal Ethics Committee of Ruijin Hospital Affiliated to Shanghai Jiao Tong University School of Medicine.

\section{Patient consent for publication}

Not applicable.

\section{Competing interests}

The authors declare that they have no competing interests.

\section{References}

1. Bray F, Ferlay J, Soerjomataram I, Siegel RL, Torre LA and Jemal A: Global cancer statistics 2018: GLOBOCAN estimates of incidence and mortality worldwide for 36 cancers in 185 countries. CA Cancer J Clin 68: 394-424, 2018.

2. Schatten H: Brief overview of prostate cancer statistics, grading, diagnosis and treatment strategies. Adv Exp Med Biol 1095: 1-14, 2018.

3. Shah ET, Upadhyaya A, Philp LK, Tang T, Skalamera D, Gunter J, Nelson CC, Williams ED and Hollier BG: Repositioning 'old' drugs for new causes: Identifying new inhibitors of prostate cancer cell migration and invasion. Clin Exp Metastasis 33: 385-399, 2016

4. Liu G, Ren F and Song Y: Upregulation of SPOCK2 inhibits the invasion and migration of prostate cancer cells by regulating the MT1-MMP/MMP2 pathway. PeerJ 7: e7163, 2019.

5. Tang C, Liu T, Wang K, Wang X, Xu S, He D and Zeng J: Transcriptional regulation of FoxM1 by HIF-1 $\alpha$ mediates hypoxia induced EMT in prostate cancer. Oncol Rep 42: 1307-1318, 2019.

6. Wang G, Zhao D, Spring DJ and DePinho RA: Genetics and biology of prostate cancer. Genes Dev 32: 1105-1140, 2018.

7. Sartor O and de Bono JS: Metastatic prostate cancer. N Engl J Med 378: 645-657, 2018.

8. Nguyen DX, Bos PD and Massagué J: Metastasis: From dissemination to organ-specific colonization. Nat Rev Cancer 9: 274-284 2009.

9. Chaffer CL and Weinberg RA: A perspective on cancer cell metastasis. Science 331: 1559-1564, 2011

10. Massagué $\mathbf{J}$ and Obenauf AC: Metastatic colonization by circulating tumour cells. Nature 529: 298-306, 2016.

11. Arpin M, Chirivino D, Naba A and Zwaenepoel I: Emerging role for ERM proteins in cell adhesion and migration. Cell Adhes Migr 5: 199-206, 2011.

12. McClatchey AI: ERM proteins. Curr Biol 22: R784-R785, 2012.

13. Ponuwei GA: A glimpse of the ERM proteins. J Biomed Sci 23: 35,2016
14. Louvet-Vallée S: ERM proteins: From cellular architecture to cell signaling. Biol Cell 92: 305-316, 2000.

15. Clucas J and Valderrama F: ERM proteins in cancer progression. J Cell Sci 128: 1253, 2015.

16. 16. McClatchey AI: ERM proteins at a glance. J Cell Sci 127: 3199-3204, 2014.

17. Kuramochi S, Moriguchi T, Kuida K, Endo J, Semba K, Nishida E and Karasuyama H: LOK is a novel mouse STE20-like protein kinase that is expressed predominantly in lymphocytes. J Biol Chem 272: 22679-22684, 1997.

18. Belkina NV, Liu Y, Hao JJ, Karasuyama H and Shaw S: LOK is a major ERM kinase in resting lymphocytes and regulates cytoskeletal rearrangement through ERM phosphorylation. Proc Natl Acad Sci USA 106: 4707-4712, 2009.

19. Endo J, Toyama-Sorimachi N, Taya C, Kuramochi-Miyagawa S, Nagata K, Kuida K, Takashi T, Yonekawa H, Yoshizawa Y, Miyasaka N, et al: Deficiency of a STE20/PAK family kinase LOK leads to the acceleration of LFA-1 clustering and cell adhesion of activated lymphocytes. FEBS Lett 468: 234-238, 2000.

20. Zhang L, Lu SY, Guo R, Ma JX, Tang LY, Shen Y, Shen CL, Lu LM, Wang ZG, Liu J, et al: Knockout of STK10 promotes the migration and invasion of cervical cancer cells. Transl Cancer Res 9: 7079-7090, 2020.

21. Kupcho K, Shultz J, Hurst R, Hartnett J, Zhou W, Machleidt T, Grailer J, Worzella T, Riss T, Lazar D, et al: A real-time, bioluminescent annexin $\mathrm{V}$ assay for the assessment of apoptosis. Apoptosis 24: 184-197, 2019.

22. Odero-Marah V, Hawsawi O, Henderson V and Sweeney J: Epithelial-Mesenchymal Transition (EMT) and Prostate Cancer. Adv Exp Med Biol 1095: 101-110, 2018.

23. Adjakly M, Ngollo M, Dagdemir A, Judes G, Pajon A, Karsli-Ceppioglu S, Penault-Llorca F, Boiteux JP, Bignon YJ, Guy L, et al: Prostate cancer: The main risk and protective factors-Epigenetic modifications. Ann Endocrinol (Paris) 76: 25-41, 2015.

24. Gloerich M,Ponsioen B, Vliem MJ,Zhang Z,Zhao J, Kooistra MR, Price LS, Ritsma L, Zwartkruis FJ, Rehmann H, et al: Spatial regulation of cyclic AMP-Epac1 signaling in cell adhesion by ERM proteins. Mol Cell Biol 30: 5421-5431, 2010.

25. Lallemand D and Arpin M: Moesin/ezrin: A specific role in cell metastasis? Pigment Cell Melanoma Res 23: 6-7, 2010.

26. Koul HK, Pal M and Koul S: Role of p38 MAP kinase signal transduction in solid tumors. Genes Cancer 4: 342-359, 2013.

27. Bradham C and McClay DR: p38 MAPK in development and cancer. Cell Cycle 5: 824-828, 2006.

28. Moriwaki K and Asahi M: Augmented TME O-GlcNAcylation promotes tumor proliferation through the inhibition of p38 MAPK. Mol Cancer Res 15: 1287-1298, 2017.

29. Krebs AM, Mitschke J, Lasierra Losada M, Schmalhofer O, Boerries M, Busch H, Boettcher M, Mougiakakos D, Reichardt W, Bronsert P, et al: The EMT-activator Zeb1 is a key factor for cell plasticity and promotes metastasis in pancreatic cancer. Nat Cell Biol 19: 518-529, 2017.

30. Fu M, Wang C, Li Z, Sakamaki T and Pestell RG: Minireview: Cyclin D1: normal and abnormal functions. Endocrinology 145: 5439-5447, 2004

31. Lee RJ, Albanese C, Stenger RJ, Watanabe G, Inghirami G, Haines GK III, Webster M, Muller WJ, Brugge JS, Davis RJ, et al: pp60(v-src) induction of cyclin D1 requires collaborative interactions between the extracellular signal-regulated kinase, p38, and Jun kinase pathways. A role for cAMP response element-binding protein and activating transcription factor-2 in pp60(v-src) signaling in breast cancer cells. J Biol Chem 274: 7341-7350, 1999.

32. Wang B, Wang Z, Han L, Gong S, Wang Y, He Z, Feng Y and Yang Z: Prognostic significance of cyclin D3 expression in malignancy patients: A meta-analysis. Cancer Cell Int 19: 158, 2019.

33. Zhang C, Wu Y, Xuan Z, Zhang S, Wang X, Hao Y, Wu J and Zhang S: p38MAPK, Rho/ROCK and PKC pathways are involved in influenza-induced cytoskeletal rearrangement and hyperpermeability in PMVEC via phosphorylating ERM. Virus Res 192: 6-15, 2014.

34. Koss M, Pfeiffer GR II, Wang Y, Thomas ST, Yerukhimovich M, Gaarde WA, Doerschuk CM and Wang Q: Ezrin/radixin/moesin proteins are phosphorylated by TNF-alpha and modulate permeability increases in human pulmonary microvascular endothelial cells. J Immunol 176: 1218-1227, 2006. 